UDC 542.61

SCOPUS CODE 1106

https://doi.org/10.36073/1512-0996-2021-2-49-56

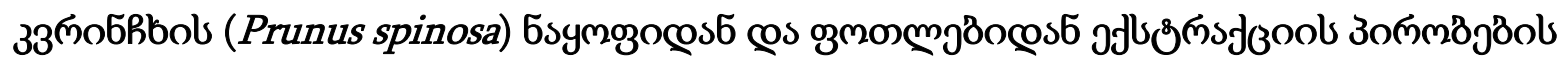

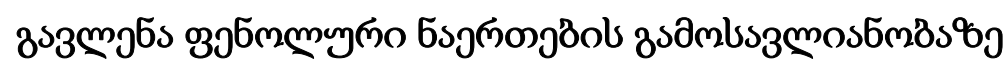

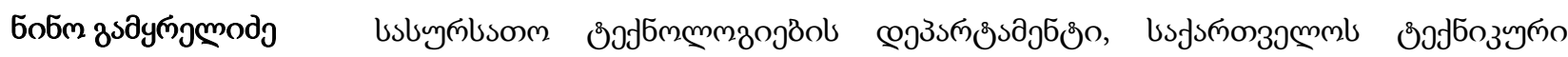

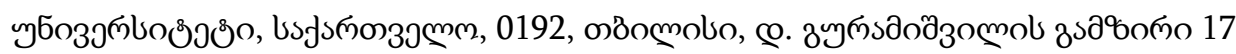 E-mail: nino.gamkrelidze@gtu.ge

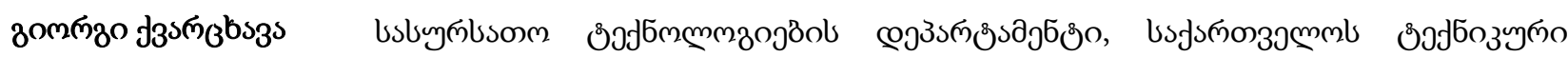

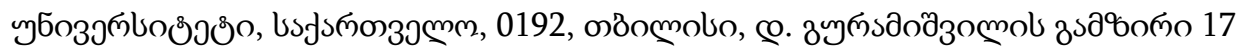 E-mail: g.kvartskhava@gtu.ge

๓วзว

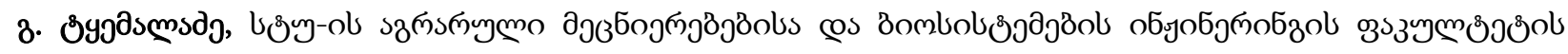

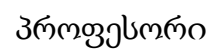

E-mail: guram.tkemaladze@yahoo.com

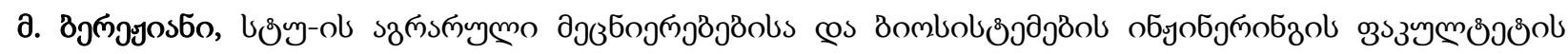

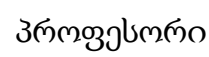

E-mail: malber@dr.com

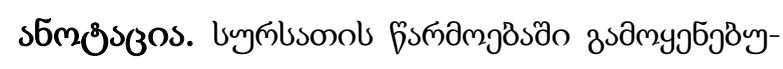

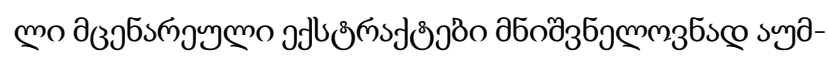

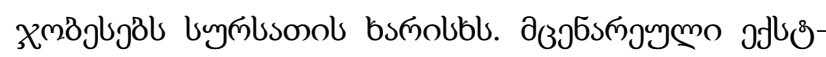

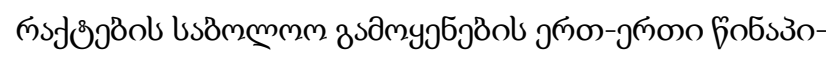

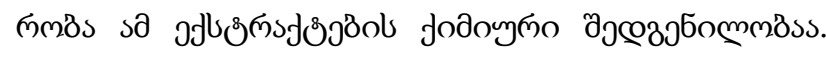

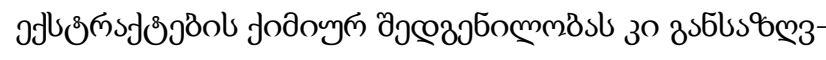

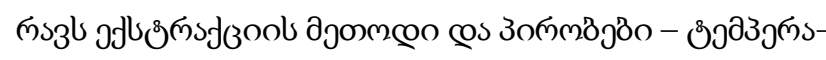

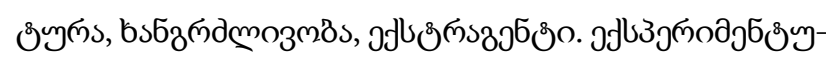

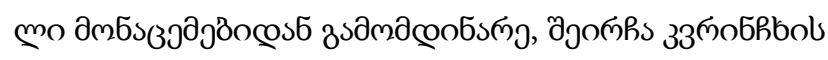

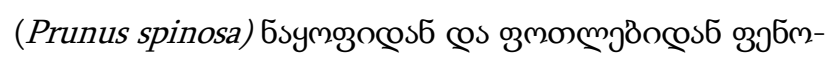

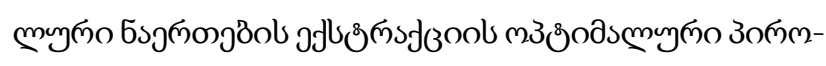

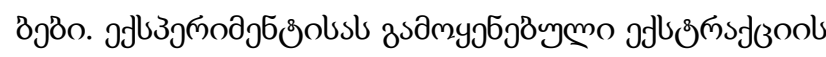

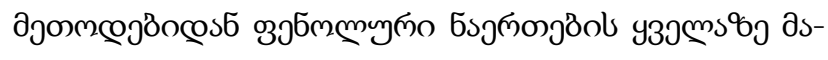

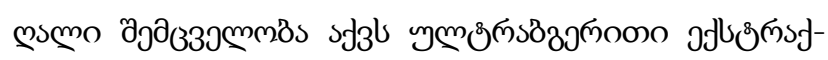
उ

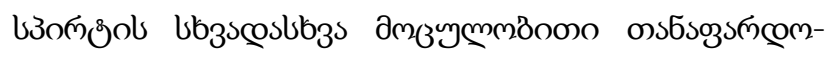

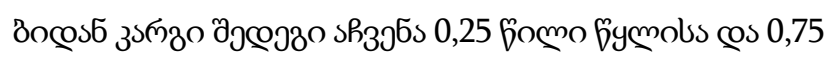

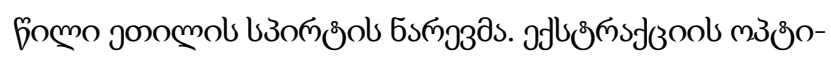

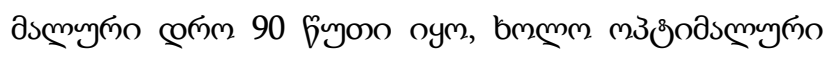

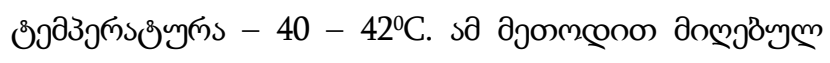

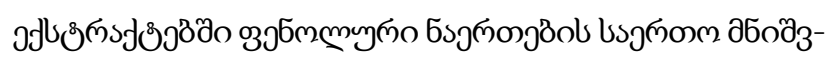

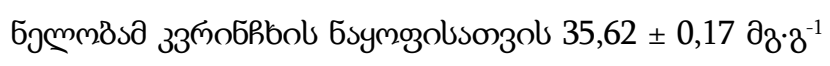

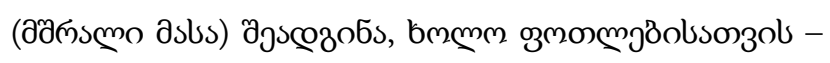

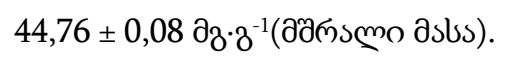




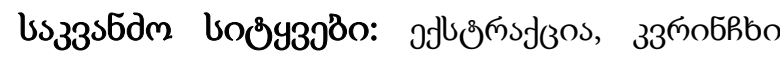

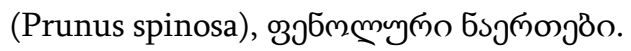

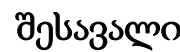

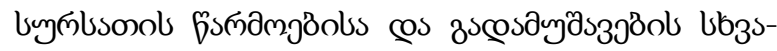

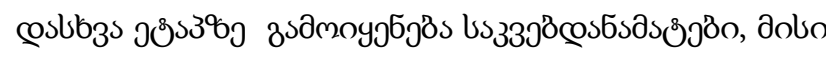

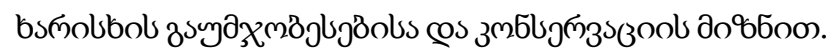

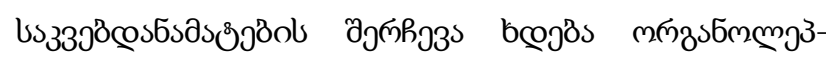

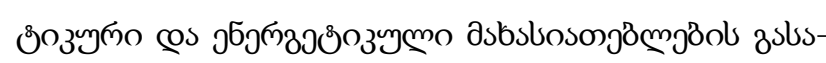

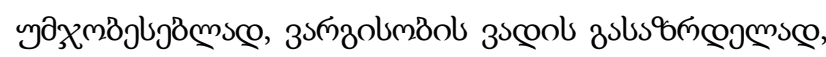

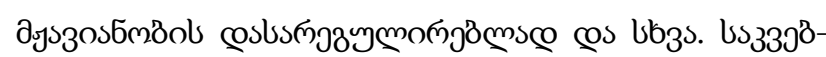

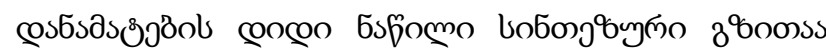

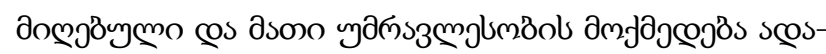

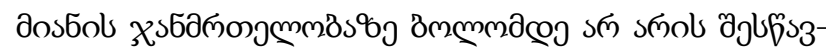

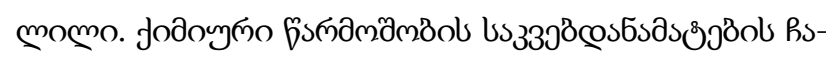

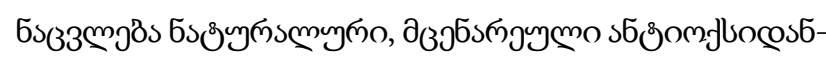

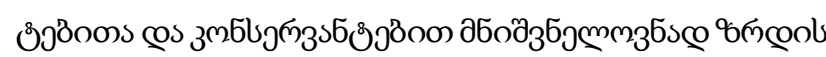

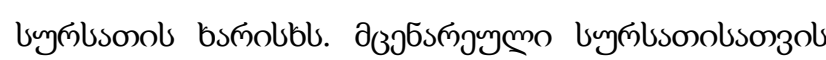

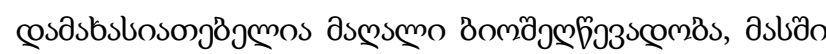

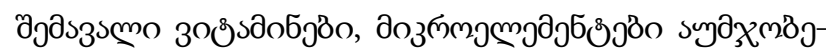

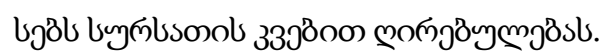

\section{domooscen 5sfomo}

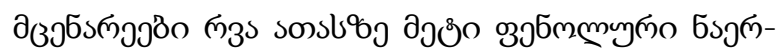

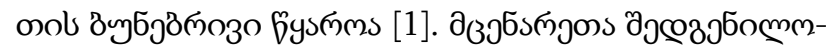

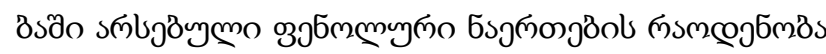

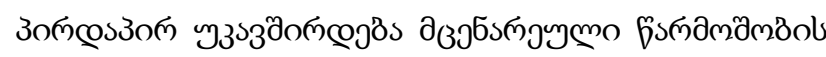

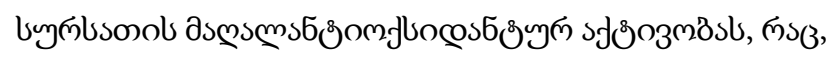

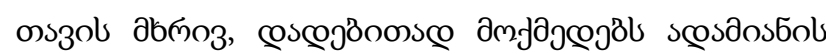

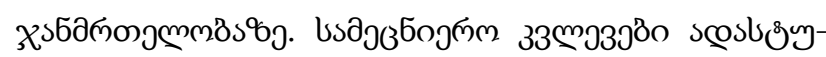

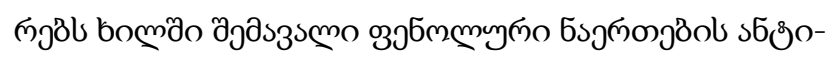

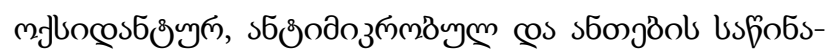

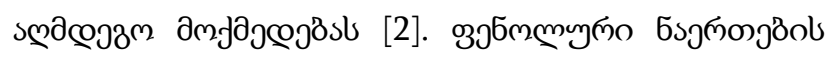

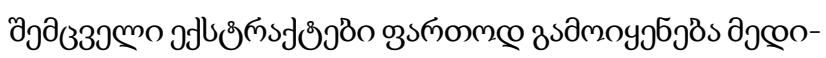

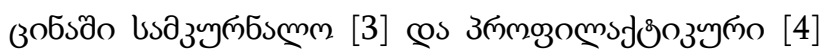
до\%бgðobsonzol.

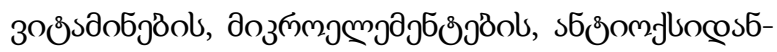

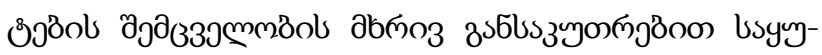

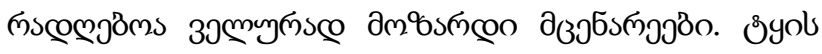

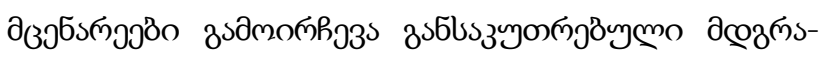

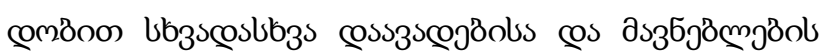

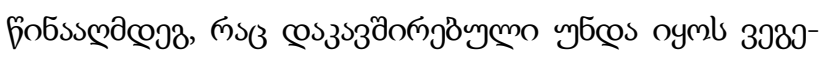

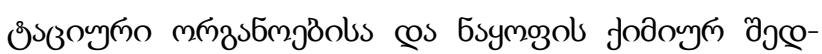

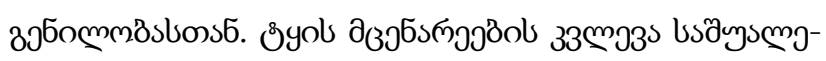

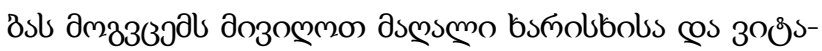

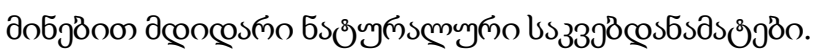

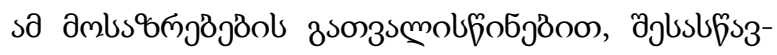

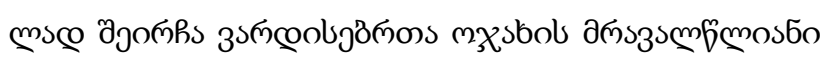

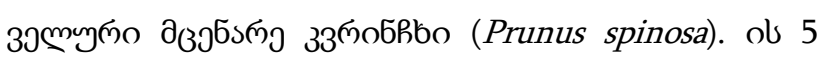

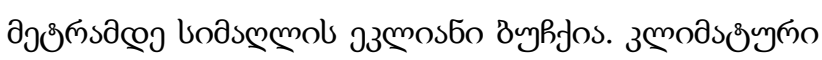

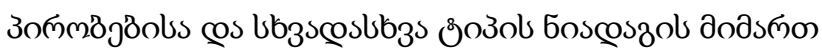

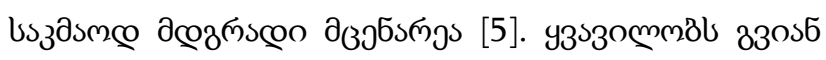

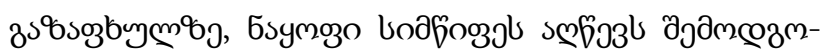

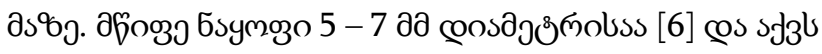

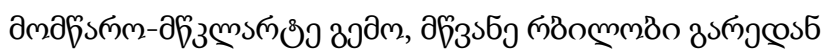

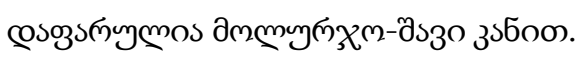

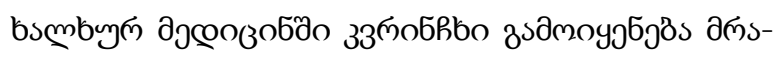

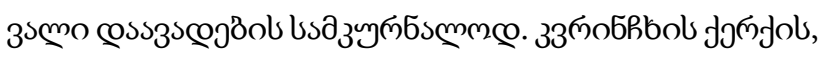

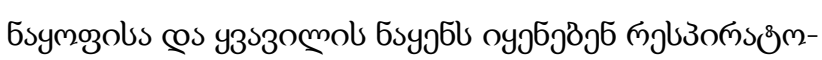

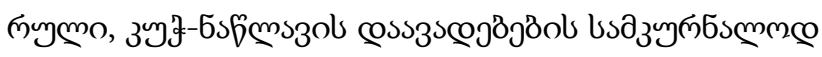

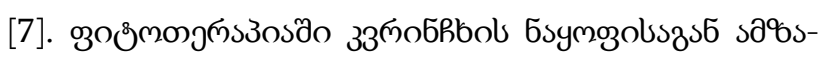

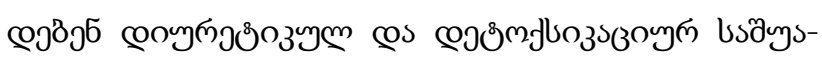

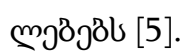

ว

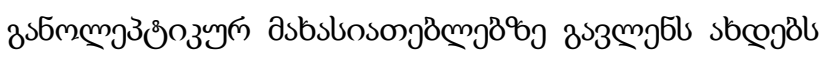

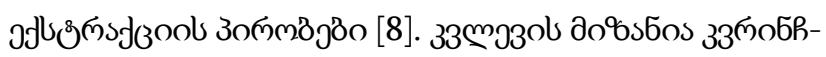




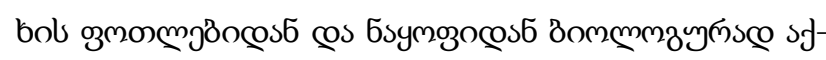

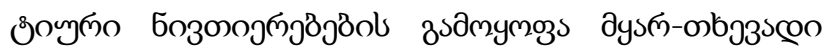

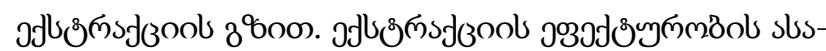

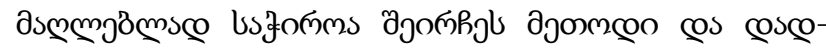

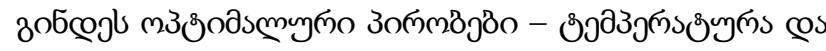

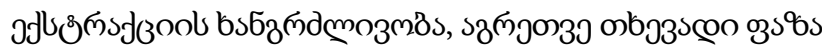

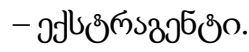

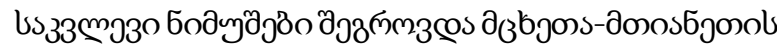
ауббозозьмов์

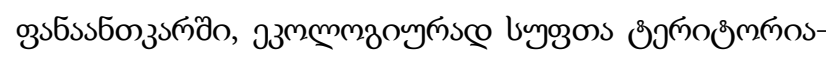

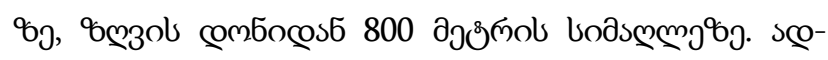

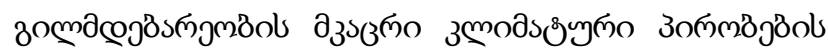

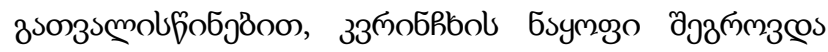

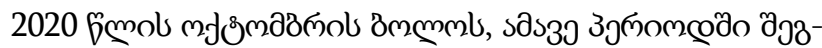

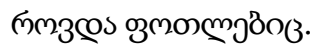

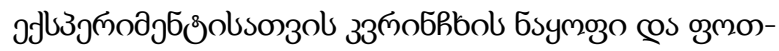

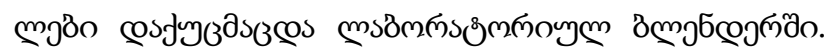

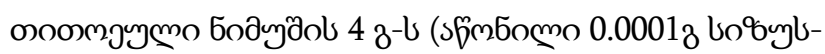

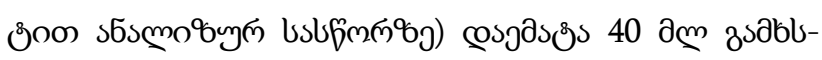

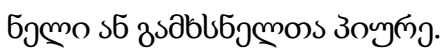

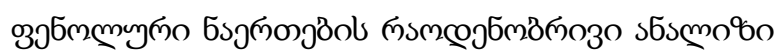

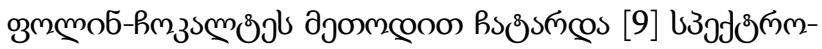

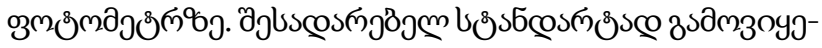

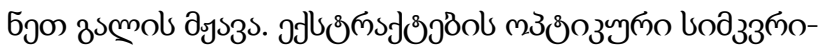

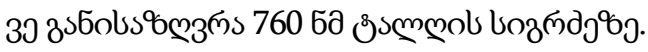

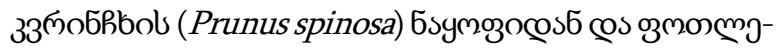

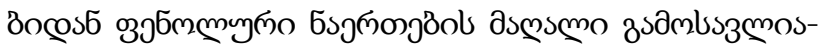

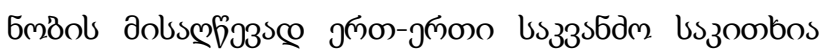

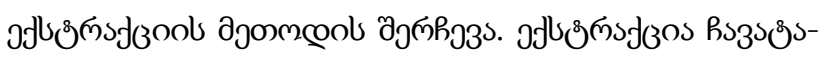

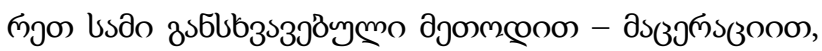

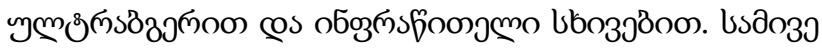

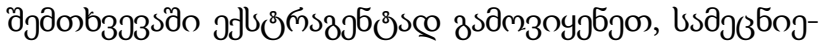

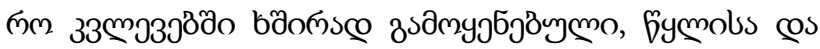

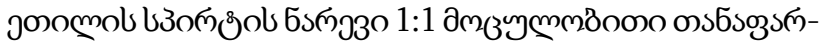

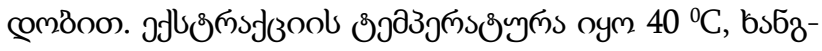

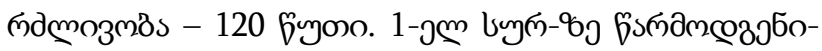

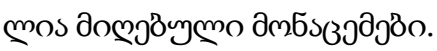

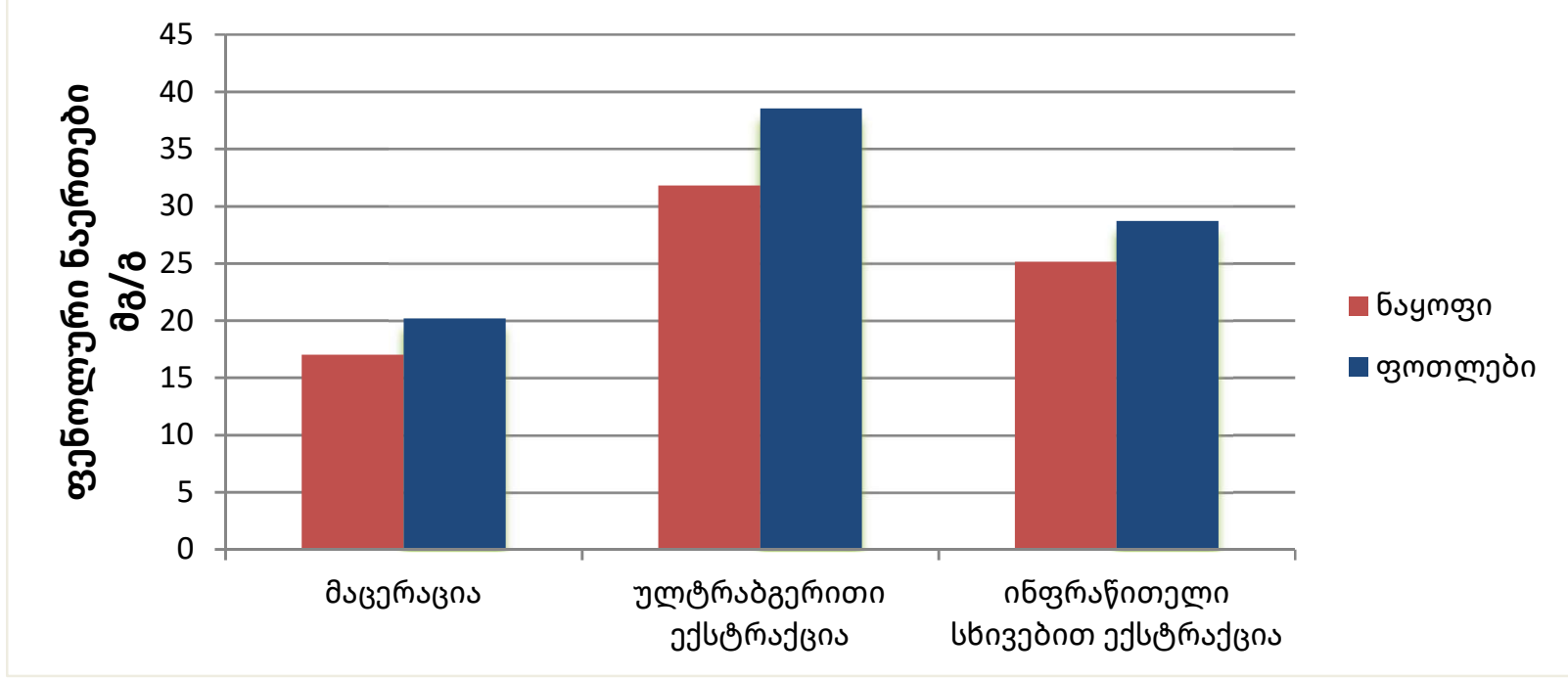

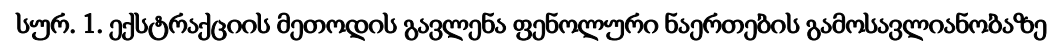




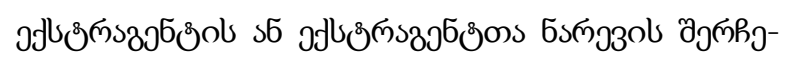

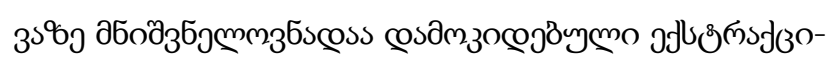

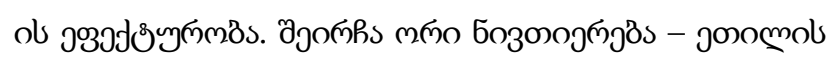

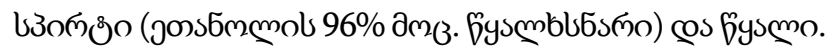

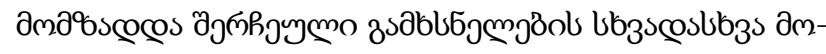

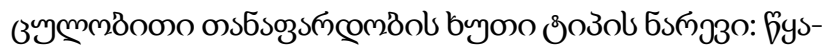

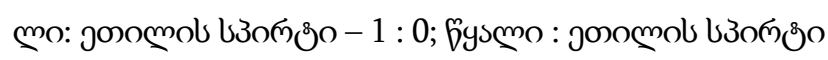

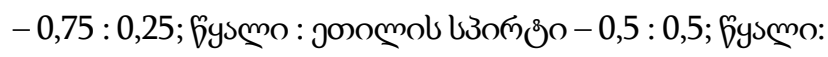

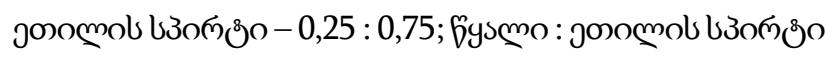

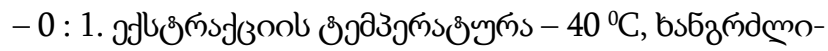
3

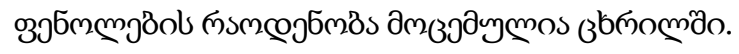

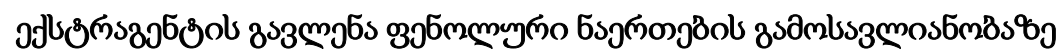

\begin{tabular}{|c|c|c|}
\hline \multirow{2}{*}{ 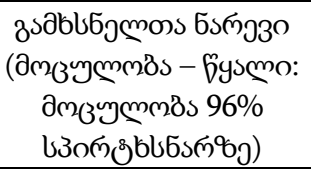 } & \multicolumn{2}{|c|}{ 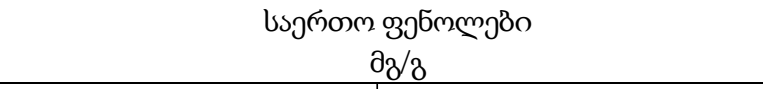 } \\
\hline & Esymogo & ஜுмому \\
\hline $\begin{array}{c}\mathrm{H}_{2} \mathrm{O}: \mathrm{C}_{2} \mathrm{H}_{5} \mathrm{OH} \\
1: 0 \\
\end{array}$ & $13,15 \pm 0,18$ & $18,31 \pm 0,21$ \\
\hline $\begin{array}{c}\mathrm{H}_{2} \mathrm{O}: \mathrm{C}_{2} \mathrm{H}_{5} \mathrm{OH} \\
0,75: 0,25 \\
\end{array}$ & $15,34 \pm 0,22$ & $20,23 \pm 0,13$ \\
\hline $\begin{array}{c}\mathrm{H}_{2} \mathrm{O}: \mathrm{C}_{2} \mathrm{H}_{5} \mathrm{OH} \\
0,50: 0,50\end{array}$ & $31,79 \pm 0,24$ & $38.54 \pm 0,09$ \\
\hline $\begin{array}{c}\mathrm{H}_{2} \mathrm{O}: \mathrm{C}_{2} \mathrm{H}_{5} \mathrm{OH} \\
0,25: 0,75\end{array}$ & $36,26 \pm 0,07$ & $45,19 \pm 0,02$ \\
\hline $\begin{array}{c}\mathrm{H}_{2} \mathrm{O}: \mathrm{C}_{2} \mathrm{H}_{5} \mathrm{OH} \\
0: 1\end{array}$ & $16,37 \pm 0,03$ & $22,63 \pm 0,14$ \\
\hline
\end{tabular}

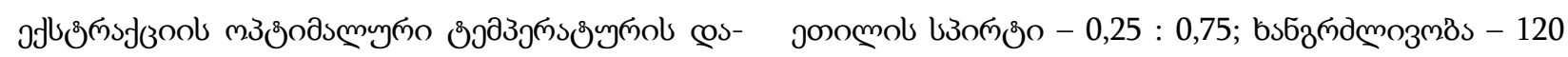

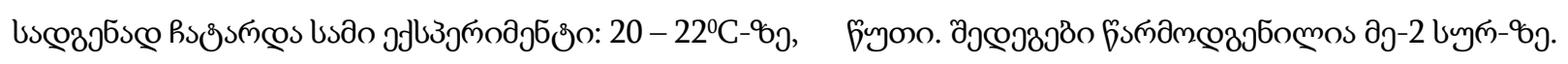

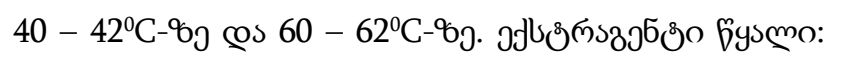

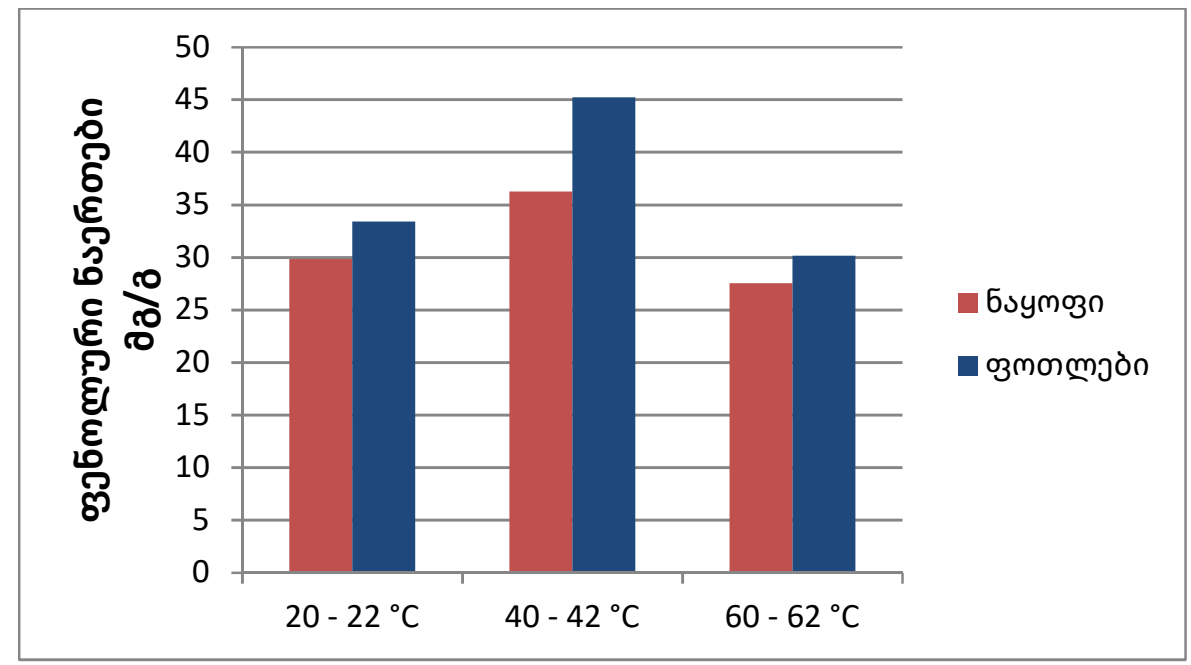

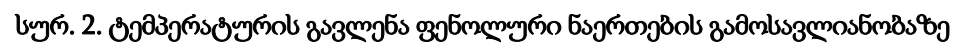




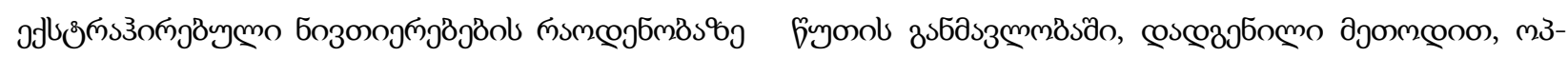

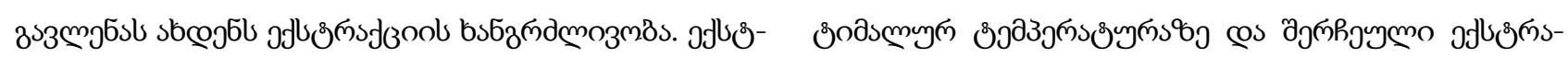

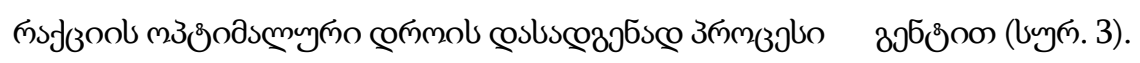

fimodsmons 30 fryonol, 60 fryonol, 90 finool cos 120

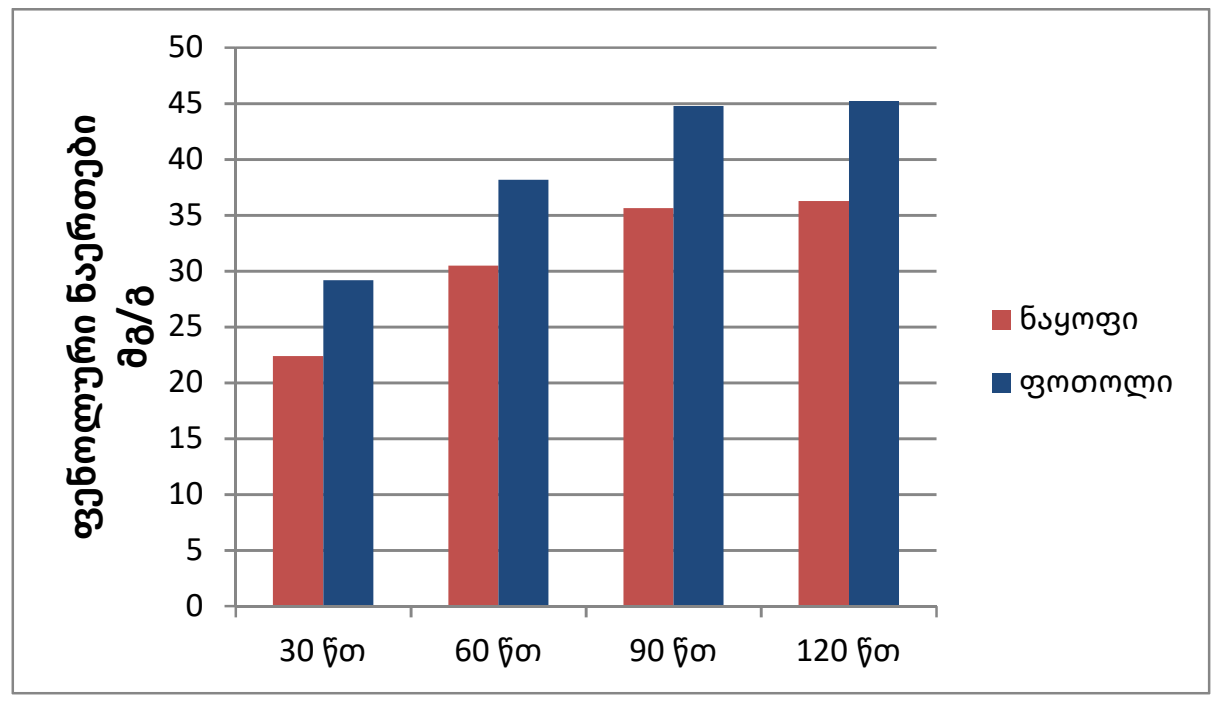

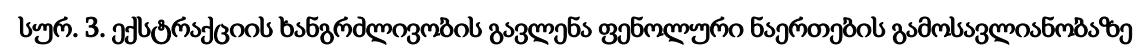

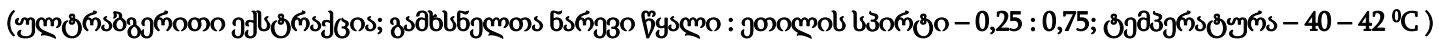

\section{$\cos 3365$}

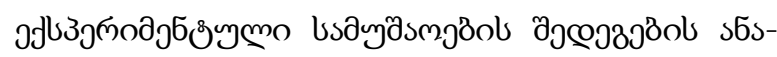

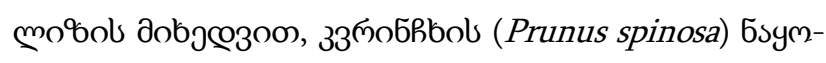

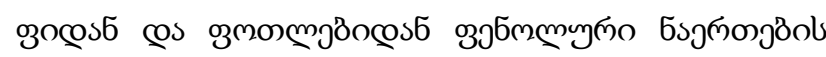

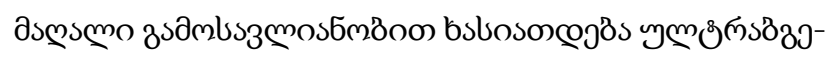

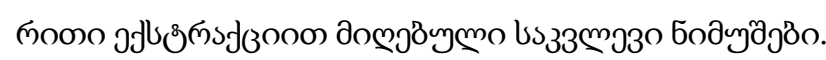

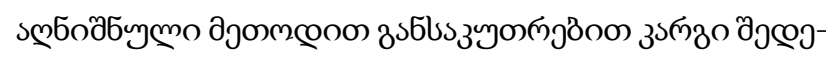

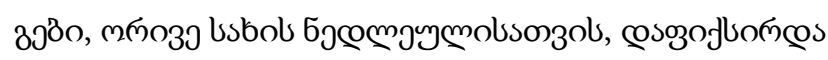
40 - 420

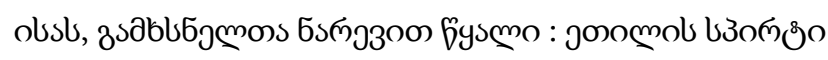

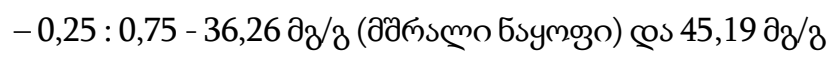

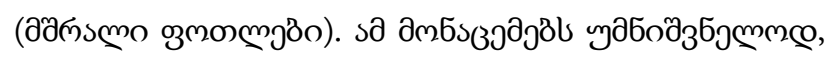

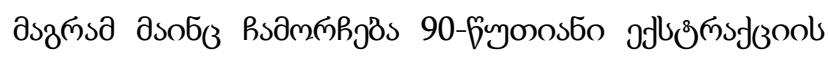

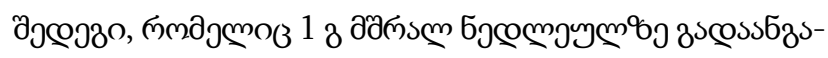

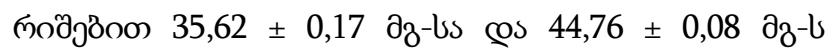

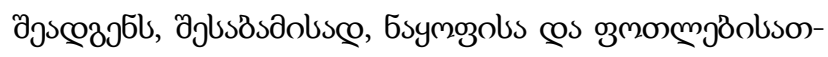

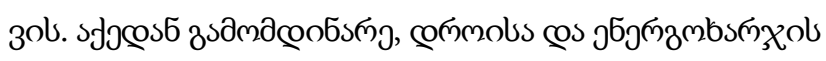
зsomзs

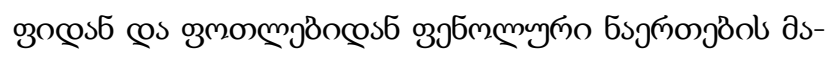

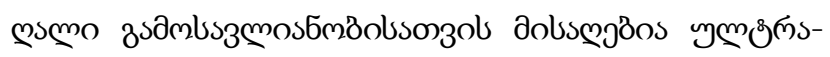

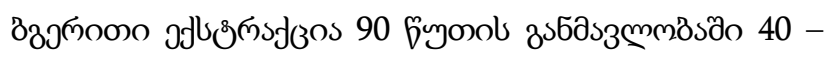

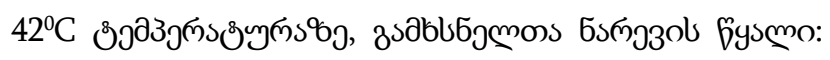

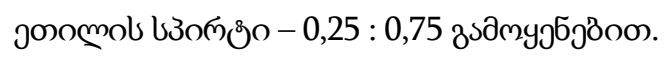




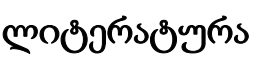

1. Crozier A., Jaganath Indu B., Clifford Michael N.- Dietary phenolics: chemistry, bioavailability and effects on health. NPR, vol.26, \#8, p. 965 - 1096, 2009.

2. Chang S.K., Alasalvar C., Shahidi F. Superfruits: Phytochemicals, antioxidant efficacies, and health effects. A comprehensive review. Critical Reviews in food Sciencwe and Nutrition, 59(10), pp. 1580 - 1604. 2019.

3. Tungmunnithum D., Thongboonyou A., Pholboon A., Yangsabai A. Flavonoids and other phenolic compounds from medicinal plants for pharmaceutical and medical aspects: An overview. Medicines. Vol.5, 93, 2018.

4. Li An-Na., Li Sha., Zhang Yu-Jie, Xu Xiang-Rong, Chen Yu-Ming, Li Hua-bin. Resources and biological activities of natural polyphenols. Nutrients, vol.6(12), p. 6020 - 6047, 2014.

5. Aparajita M., Juan Pedro M., Itziar A. Population genetic analysis of European Prunusspinosa (Rosaceae)using chloroplast DNA markers, American Journal of Botany, vol. 89, pp. 1223-1228, 2002.

6. Fraternale D., Giamperi L., Bucchini A., Piero S., Paolillo M., Ricci D. Prunusspinosa Fresh Fruit Jiuce: Antioxidant Activity in Cell-free and Cellular Systems. NPC, vol. 4, № 12, pp. 1665 - 1670, 2009.

7. Khidasheli Sh., Papunidze V. Medical plants forest Georgian. Publishing house "Soviet Adjara", Batumi, 1985 (in Georgian).

8. Terletskaya V., Rubanka E., Zinchenko I. Influence of technological factors on the process of black chokeberry extraction. Technique and technology of food production. №4, pp.127 - 131, 2013 (in Russian).

9. Singleton V.L., Rossi J.A. Colorimetry of total phenolics with phosphorrmolybdic-phosphotungstic acid reagents. Am.J.Enol.Viticult.16, pp. 144 - 158. 1965. 
UDC 542.61

SCOPUS CODE 1106

https://doi.org/10.36073/1512-0996-2021-2-49-56

\title{
The Influence of the Extraction Conditions of Blackthorn (Prunus spinosa) Fruits and Leaves on the Yield of Phenolic Compounds
}

Nino Gamkrelidze Department of Food Technology, Georgian Technical University, Georgia, 0192, Tbilisi, 17 D. Guramishvili Str.

E-mail: nino.gamkrelidze@gtu.ge

Giorgi Kvartskava Department of Food Technology, Georgian Technical University, 17 D. Guramishvili Str., 0192, Tbilisi, Georgia

E-mail: g.kvartskhava@gtu.ge

\section{Reviewers:}

G. Tkemaladze, Professor, Faculty of Agricultural Science and Biosystems Engineering, GTU

E-mail: guram.tkemaladze@yahoo.com

M. Berejiani, Professor, Faculty of Agricultural Science and Biosystems Engineering, GTU

E-mail: malber@dr.com

\begin{abstract}
Plant extracts used in food production significantly improve the quality of food. Chemical composition is the main prerequisites for the final use of plant extracts. The chemical composition of the extracts is affected by the extraction method and conditions - temperature, duration and solvent. On the basis of experimental data optimal conditions for the extraction of phenolic compounds from the fruits and leaves of blackthorn (Prunus spinosa) were selected. Among the extraction methods used in the experiment, the highest content of phenolic compounds in obtained extracts was observed during the ultrasonic extraction. A mixture of 0.25 parts of water and 0.75 parts of $96 \%$ ethanol showed best results from different volume ratios of water and ethanol. The optimal extraction time was 90 minutes. And the optimum temperature is $45^{\circ} \mathrm{C}$. The total content of phenolic compounds in the extracts obtained by the abovementioned method amounted is $35.62 \pm 0.17 \mathrm{mg} \cdot \mathrm{g}^{-1}$ (dry weight) for blackthorn fruits and $44,76 \pm$ $0.08 \mathrm{mg} \cdot \mathrm{g}^{-1}$ (dry weight) for the leaves.
\end{abstract}

Key words: blackthorn (Prunus spinosa); extraction; phenolic compounds. 
UDC 542.61

SCOPUS CODE 1106

https://doi.org/10.36073/1512-0996-2021-2-49-56

\title{
Влияние условий экстракции на выход фенольных соединений из плодов и листьев терновника (Prunus spinosa)
}

\author{
Нино Гамкрелидзе Департамент пищевой технологии, Грузинский технический университет, Грузия, \\ 0192, Пр. Д. Гурамишвили 17 \\ E-mail: nino.gamkrelidze@gtu.ge \\ Георгий Кварцхава Департамент пищевой технологии, Грузинский технический университет, Грузия, \\ 0192, Пр. Д.Гурамишвили 17 \\ E-mail: g.kvartskhava@gtu.ge
}

\section{Рецензенты:}

Г. Ткемаладзе, профессор факультета аграрных наук и инжинеринга биосистем ГТУ

E-mail: guram.tkemaladze@yahoo.com

М. Бережиани, профессор факультета аграрных наук и инжинеринга биосистем ГТУ

E-mail: malber@dr.com

Аннотация. Растительные экстракты, используемые в пищевом производстве, значительно улучшают качество продуктов питания. Химический состав является основной предпосылкой для конечного использования растительных экстрактов. Химический состав экстрактов определяет способ и условия экстракции температура, продолжительность и экстрагент. На основании экспериментальных данных были выбраны оптимальные условия извлечения фенольных соединений из плодов и листьев терновника (Prunus spinosa). Среди методов экстракции, использованных в эксперименте, наибольшее содержание фенольных соединений в полученных экстрактах наблюдалось при ультразвуковой экстракции. При различных объемных соотношениях воды и этанола наилучшие результаты показала смесь 0,25 части воды и 0,75 части 96 \%-ого этанола. Оптимальное время экстракции составляло 90 минут. А оптимальная температура $-40-42{ }^{\circ} \mathrm{C}$. Общее содержание фенольных соединений в экстрактах, полученных указанным способом для плодов терновника составило $35,62 \pm 0,17$ мг $\cdot \Gamma^{-1}$ (на сухой вес) и $44,76 \pm 0,08$ мг·г-1 (на сухой вес) для листьев.

Ключевые слова: терновник (Prunus spinosa); фенольные соединения; экстракция.

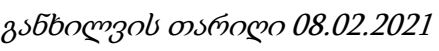

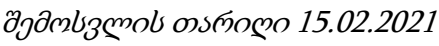

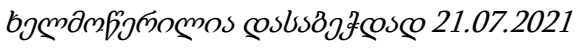

